\title{
Simulation of Autonomous Mobility of Connected Vehicles in the Context of Real Conditions - a Case Study
}

\author{
Jiří Hanzl $^{1 *}$, Patrik Gross $^{1}$, Ladislav Bartuška ${ }^{1}$ and Jan Pečman ${ }^{1}$ \\ ${ }^{1}$ Institute of Technology and Business in Ceske Budejovice, Faculty of Technology, Department of \\ Transport and Logistics, 37001 Ceske Budejovice, Czech Republic; Email: hanzl@mail.vstecb.cz, \\ gross@mail.vstecb.cz,bartuska@mail.vstecb.cz,pecman@mail.vstecb.cz
}

\section{*Corresponding Author: Jiří Hanzl}

Received: 10 November 2021; Revised: 12 November 2021; Accepted: 25 November 2021;

Published: 30 November 2021

\begin{abstract}
By designing road infrastructure, it is necessary to adapt the real situation to current development trends and respond accordingly to the intensity of traffic on the transport network. The development of the traffic situation is generally very dynamic, difficult to predict and influenced by a number of other factors. Modern technologies enable adaptive traffic flow management based on the sharing and evaluation of traffic information obtained in real time from traffic monitoring systems or even from vehicles as such (e.g. thanks to "Connected Vehicles" technology). The article first carries out a literature review of professional literature and scientific articles dealing with the issue of autonomous mobility and autonomous management of transport processes. That is followed by a description and creation of own algorithm for autonomous control of vehicles at the level crossing, including description of used data, methods and proposed solutions. Finally, the developed method (algorithm) is tested by the Anylogic simulation program in a real environment, as a case study of autonomous vehicle decision-making at the level crossing.
\end{abstract}

Keywords: Autonomous mobility, connected vehicles, simulation, traffic density, traffic flow

\section{Introduction}

At present, autonomous mobility is a big topic and a future trend, which cannot be viewed only to a limited extent in terms of the technology of its own autonomous vehicle control, but in terms of the management of the transport system as a whole. Already in the 1960s, several projects were launched, whose vision was to design a vehicle that will not need a person to drive. Unfortunately, most of these projects were gradually terminated, partly due to insufficient results and partly due to their high financial demands. From time immemorial, however, the fact that the human factor, although irreplaceable in transport management processes, is at the same time the weakest link in the whole system of ensuring the safety of transport infrastructure. For this reason, even today there is an effort 
to gradually move to an autonomous way of driving vehicles, which both eliminates human errors and increases the safety, reliability and efficiency of the transport infrastructure. Particularly in large cities and conurbations, the autonomous vehicle management system is intended to help address the often limited conditions of street space, congested branches of level crossings and mitigate the effects of traffic restrictions on the fluidity and safety of road traffic on detours and parallel roads.

In the following chapters of this article, a literature review of professional literature and scientific articles dealing with the issue of autonomous mobility and autonomous management of transport processes is first performed. That is followed by a description and creation of its own algorithm for autonomous control of vehicles at the level crossing, including a description of the data used, methods and proposed solutions. Last but not least, the developed method (algorithm) is tested by the Anylogic simulation program in the context of a real environment, as a case study of autonomous vehicle decision-making at a level crossing.

\section{Literature Review}

Traffic signal controls play an important role in regulating vehicular flow at road intersections. Traditional systems are not capable of adjusting the timing pattern in accordance with vehicular demand. Hence it is necessary to develop dynamic systems that can adjust the timing patterns according to traffic demand, and autonomous vehicles should be involved in these dynamic systems.

Gulai et al. [1] have tested the idea of using connected vehicle technology to improve the efficiency of intersections controlled with traffic lights. In their research they developed algorithms to minimize total delay or number of stops. A simulation with algorithms showed a significant delay reduction with increasing penetration rate under the proposed algorithms. Other authors and researchers came with their algorithms and adaptive traffic control systems based on car-to-car communication for improving dynamic traffic lights control system at intersections, with all leading to increased efficiency of traffic control [2-4]. The simulations also showed that the data convergence time and the communication delay between vehicles and traffic signals do not compromise the efficiency of the system.

Furthermore, other researchers focused their work on using agent-based models to optimize traffic signal control in a connected vehicle environment [5]. The models have an advantage in studying several agents specified at various scenarios. Kari et al. [6] proposed an agent-based online adaptive traffic signal control (ATSC) based on connected vehicle technology. Their proposed framework consists of two types of agents: vehicle agents and signal controller agents. Each vehicle agent communicates with others and signal controller agents transmit the estimation or prediction of its key statistics. The statistics gathered by signal controller agents can then be aggregated into some critical metrics at the lane to support the signal control. 
While the majority of above papers optimize traffic performance, the agent-based models can be also used for optimizing multi-intersection signal control. Ezawa et al. [7] proposed an adaptive traffic signal control based on vehicle route sharing. It lies in sharing the position and path information, and the route sharing information was used for calculating expected traffic congestion. Xiang et al. [8] presented a multi-agent-based control method for an integrated network of adaptive traffic signal controllers under V2I communication environment.

\section{Data and Methods}

When designing road infrastructure, it is necessary to adapt the real situation to current development trends and respond accordingly to the increase in traffic intensity, crisis situation or changes in traffic not only in the urban environment but also on the outskirts or completely outside the built-up area. The development of the traffic situation is generally very dynamic, difficult to predict and influenced by a large number of other factors directly and indirectly related to the current situation on the transport network or the transport system as such.

Modern technologies today enable adaptive traffic flow management based on the sharing and evaluation of traffic information obtained in real time from traffic monitoring systems or even from vehicles as such (the already mentioned "Connected Vehicles" technology). Before the actual introduction and direct intervention of modern traffic management systems in the current situation on the transport network, it is appropriate to first use tools for modeling, simulation and subsequent analysis of data obtained in comparison with the real form and requirements set for solving a specific traffic situation. This simulation can serve not only as a tool to verify functional principles, solution variants or possible scenarios, but also as a platform for simulating real events from the past using data obtained from the current form of traffic flow: i.e. a simulation of an event under current conditions and traffic system [9]. Ultimately, using such modern methods for the transport user himself/herself increases quality, accessibility and user comfort, while the subsequent optimization of traffic flows generally increases the economy, efficiency and safety of transport, which will further reduce negative environmental and social impacts.

\subsection{Simulations and Models}

The use of models and simulations is nothing new in scientific practice. The advent of information technology is directly linked to the use of simulation technologies. At the current pace of development, new and more complex methods, technologies or procedures for imitating real systems in a virtual environment are emerging. Thus, in general, a simulation can be defined as an attempt to mimic a real system, state, or process in order to examine relevant stochastic or deterministic elements or properties of the system through experiment. The model is therefore always based on the 
simplification or abstraction of reality. It is never possible to create a model that perfectly corresponds to the real system in all its details [10]. If such a model could be created, it would not be possible to use it for practical reasons, as the complexity of the model increases the demands on computer computing power, increases the time required to perform the simulation and reduces the explanatory power due to excessive complexity of subsequent output analysis. The usefulness of the model therefore lies primarily in simplifying or reducing the complexity of the modelled object in order to achieve a higher informative value of the obtained outputs. At the same time, however, comes the need to evaluate to what extent and what elements of the model can be simplified, so as not to affect or reduce the quality of the monitored outputs [11].

In the context of transport, it is therefore a general improvement of the design or planning process and the subsequent easier management of the transport system or its elements. Although the first use of traffic simulations can be traced back to the second half of the twentieth century, it is now an integral part of any traffic planning and construction of new transport infrastructure. A significant advantage of performing traffic simulations in a virtual environment is the ability to test various scenarios in real or simulation time without the need to intervene in the real situation on the transport network [12,13]. Real-world testing is usually not possible for practical, time, safety or financial reasons, even if the transport section is available. The main benefit of traffic simulations lies mainly in the possibility of using this technology in cases where other methods do not achieve the required accuracy or are not able to imitate the situation [14]. Simulations can be divided according to several criteria:

- $\quad$ static simulation - the state of the simulation does not change over time, so it is less demanding on computing power, it can better capture more complex systems

- dynamic simulation - the state of the simulation changes over time, so it is necessary to recalculate all simulated parameters during the simulation; this increases the demands on computing power, but it also increases the ability to mimic real systems that change over time transport systems.

Next, simulations can be divided according to the extent to which the simulated system is described:

- macroscopic simulation - this simulation uses a lower level of detail, the system is simplified to basic factors, allows the simulation of large systems where the real interaction of individual agents is not essential,

- mesoscopic simulation - this simulation is a transition between macroscopic and microscopic simulation, it can contain elements of both categories with regard to a specific situation; these are simulations that depict individual elements of the system, but their interactions are limited,

- microscopic simulation - this simulation shows in detail the individual elements of the system as well as their mutual interactions; in the context of traffic flow simulation, an example can be 
given when individual vehicles are in a collision path and mutual interaction is required, i.e. a change of direction or braking.

Depending on whether the simulation uses probability or not, it is divided into:

- stochastic simulation - random numbers and probability distributions are used in the simulation

- deterministic simulation - during the simulation, the interactions between individual elements in the system are unambiguously determined by the input data.

The creation of the simulation itself involves a number of follow-up tasks, which consist in an analysis of the simulated system. It is about determining the specific elements of the system that need to be included in the simulation, then it is necessary to evaluate what will be the form of input data with respect to the required outputs. When simulating a traffic flow, it is therefore necessary to have not only values of traffic intensities, but also the spatial arrangement of the road. In case of traffic junction simulation, it is also necessary to include data concerning, for example, the control of an intersection or the speed limit on a given road section [15]. Traffic intensity values can be obtained by simple observation, measurement through traffic monitoring technologies or on the basis of design or prospective traffic intensities calculated from historical data or from the theoretical permeability of a given road. The next step is constructing the model itself, which is influenced mainly by the platform used, or the type of simulation software, the available computing power of the computer and the requirements for output data. After the simulation or during the simulation, the analysis of the obtained information, its evaluation and often also repeated testing take place. When using simulation software, there are a large number of tools and methods that in many cases have similar elements or processes, but use different programming languages and provide different types of output.

\subsection{Simulation System AnyLogic}

To create a simulation model in this case study, the AnyLogic program from the multinational company of the same name was used, which started developing it in 1992. It is a simulation software using the Java programming language, which supports three basic simulation methods.

- Discrete event simulation (DES). When using the simulation of discrete events, the change in time does not take place continuously, but changes abruptly at specific times, or when an event occurs, such as an element entering the system or a change in the state of a system element after interaction. This simulation method is typical for simulating queue theory and derived systems [16].

- System dynamics simulation (SDS). In contrast to the simulation of discrete events, system dynamics uses a continuous view of the flow of time. The simulation model is therefore evaluated at all times and adjusts the individual variable values. It is used mainly in solving logistics flows, where feedback systems are manifested. 
- Agent-based simulation (ABS). Simulations using agents, or also agent simulations, differ from ther methods in the overall approach to simulation. While in the previous methods it is necessary to enter the properties of the solved elements, their mutual interactions and properties within the model, in the simulation using agents it is not necessary to enter the exact behaviour of the simulated elements. The simulation is created "from below" - first it is necessary to adjust the properties and behaviour of the basic elements of the simulated system. The final form of the simulation is determined by the behaviour of individual agents, which represent the basic elements of the investigated system. In case of traffic flow simulation, these are individual participants in the traffic flow, i.e. vehicles or pedestrians.

The AnyLogic simulation program contains pre-prepared libraries. For the purpose of traffic simulation, a road traffic library is pre-prepared, which enables the simulation of traffic flow [17]. Individual libraries can be used within one simulation model. The Road Traffic Library allows one to model, simulate and then visualize road traffic. This library supports a detailed physical model of vehicle movement. The AnyLogic program is therefore suitable for modelling motorway traffic, street traffic, local transport in production plants, car parks or other systems with vehicles, roads and lanes [18-20]. The simulation can then be displayed in 3D. In the simulation, individual cars calculate the shortest route using Dijkstra's algorithm, which does not take into account the density of traffic on the roads and always calculates only the shortest route. The library of road traffic can be combined with the library of modelling of processes, pedestrians and railway transport. Thanks to this, it is possible to create vehicle operation models with models of trucks, cranes, ships, trains, passengers, production or business processes, etc.

\section{Results}

To simulate autonomous mobility and the "Connected Vehicles" system, it is appropriate to use the integrated function of the program - Optimization Experiment. To create an optimization experiment, it must be first decided what to optimize. Within this case study, it is basically a matter of creating a system of adaptive control of the intersection of A. Barcala and M. Horáková streets in České Budějovice. To set up the experiment, it was first necessary to parameterize the time of the individual light phases of the intersection arms. In this case, there were four parameters that determine the time of each light phase of the intersection. An input value of $30 \mathrm{~s}$ was set for these parameters and then these parameters were entered into the AnyLogic program in the field for the value of the duration of individual phases in the "trafficLight" block (see Fig. 1). 

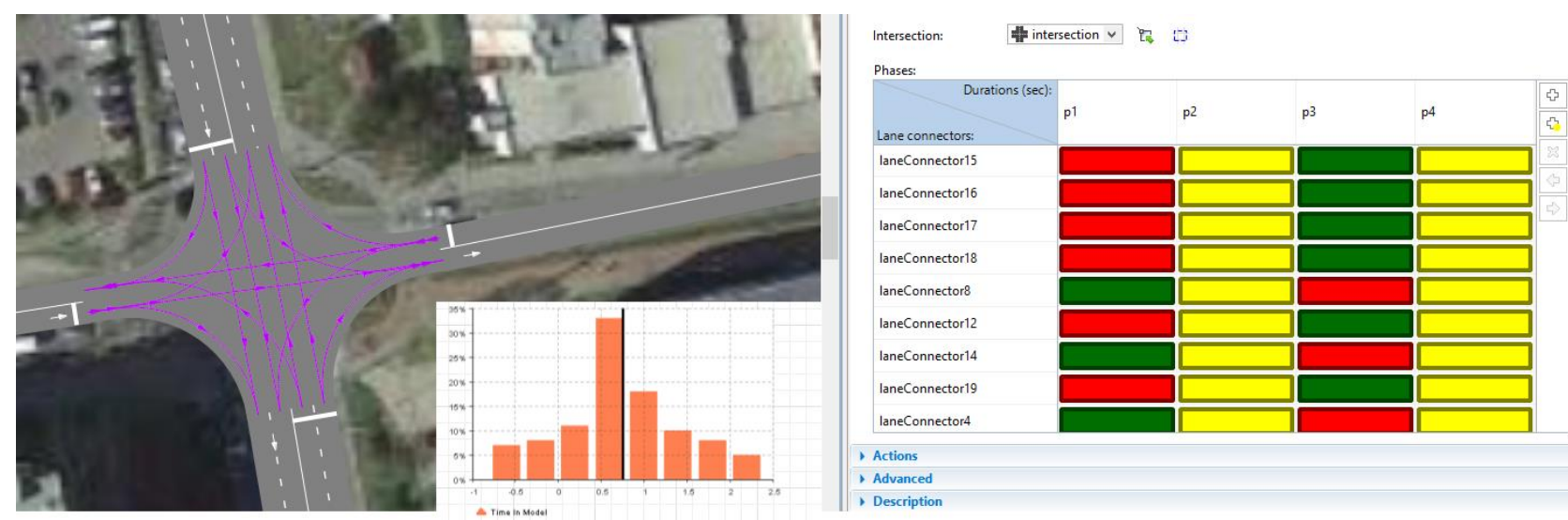

Fig. 1 Setting the parameterization of the phases of adaptive control of a traffic light

Source: authors

After selecting the optimization, the experiment settings will be displayed. The aim of this study was to minimize the average time spent in the simulation. After entering the command "root.timeInModel.mean" you can it is possible to set the parameter type to discrete, specify the range and the simulation step in which the range will change. Furthermore, it is possible to set the range of computer performance that is used to perform the optimization and, last but not least, the number of iterations that will be performed within the simulation. The last step was to create a basic (default) user interface and run the whole experiment.

\section{CV_krizovatka : Optimization}

\begin{tabular}{lr|r} 
& Current & Best \\
\hline Iterations completed: & 510 & 191 \\
Objective: $\downarrow$ & 980.994 & 118.63 \\
Parameters & & Copy best \\
\hline D1 & 35 & 5 \\
D2 & 90 & 10 \\
D3 & 15 & 90 \\
D4 & 80 & 5
\end{tabular}

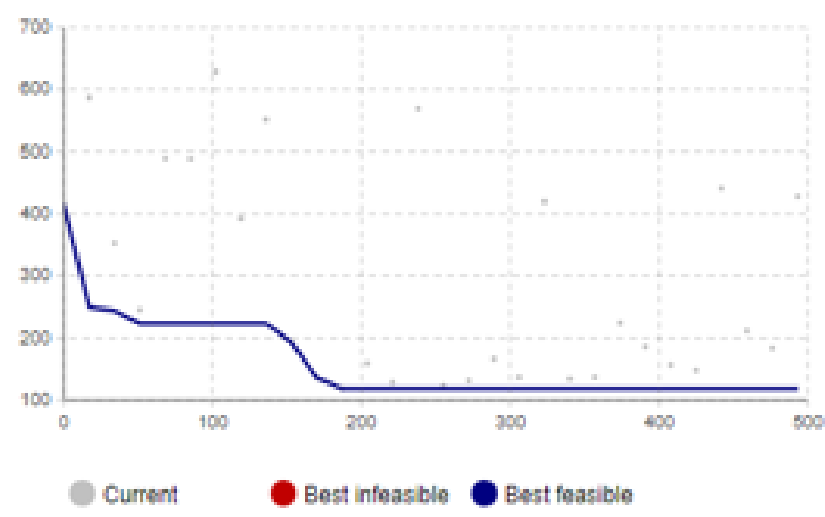

Fig. 2 Optimization experiment. Source: authors

The graph in Fig. 2 shows the actual course of optimization, where the $\mathrm{x}$-axis represents the number of simulations and the y-axis shows the goal of optimization and its best and worst value. After completing the optimization process, it is possible to copy the best achieved values and then paste them into the parameter values. In this step, however, it is necessary to consider the fact that the created algorithm takes into account only those vehicles that have left the simulation (or passed the entire simulated section). In case of blocking the traffic section, it is first necessary to limit the 
number of generated agents so that the blocking does not occur (vehicles do not leave the simulation and their time spent in the simulation is not calculated). Subsequently, the number of vehicles can be increased until the traffic section is blocked again.

\subsection{Determination of the Optimal Route According to the Number of Vehicles}

The "selectOutput" block was used in this case study to simulate the determination of the optimal route according to the number of vehicles on a given traffic section. This block routes incoming agents to one of the output ports depending on a probabilistic or deterministic condition. This condition may depend on the specific type of agent as well as external factors. In this case, the "If Condidion Is True" function was used. Using the "size" command, it is possible in the AnyLogic software to refer to the number of vehicles or a specific type of agent in a specific "carMoveTo" block (or road section). Subsequently, it is possible to set conditions using mathematical logic operators.

Therefore, for this case study, a representative model of the road was first created (see Fig. 3), where the vehicles try to gradually drive from:

- the upper left corner of the transport network to the lower right corner

- the upper right corner to the lower left corner

- the lower left corner to the upper right corner

- the lower right corner to the upper left corner.

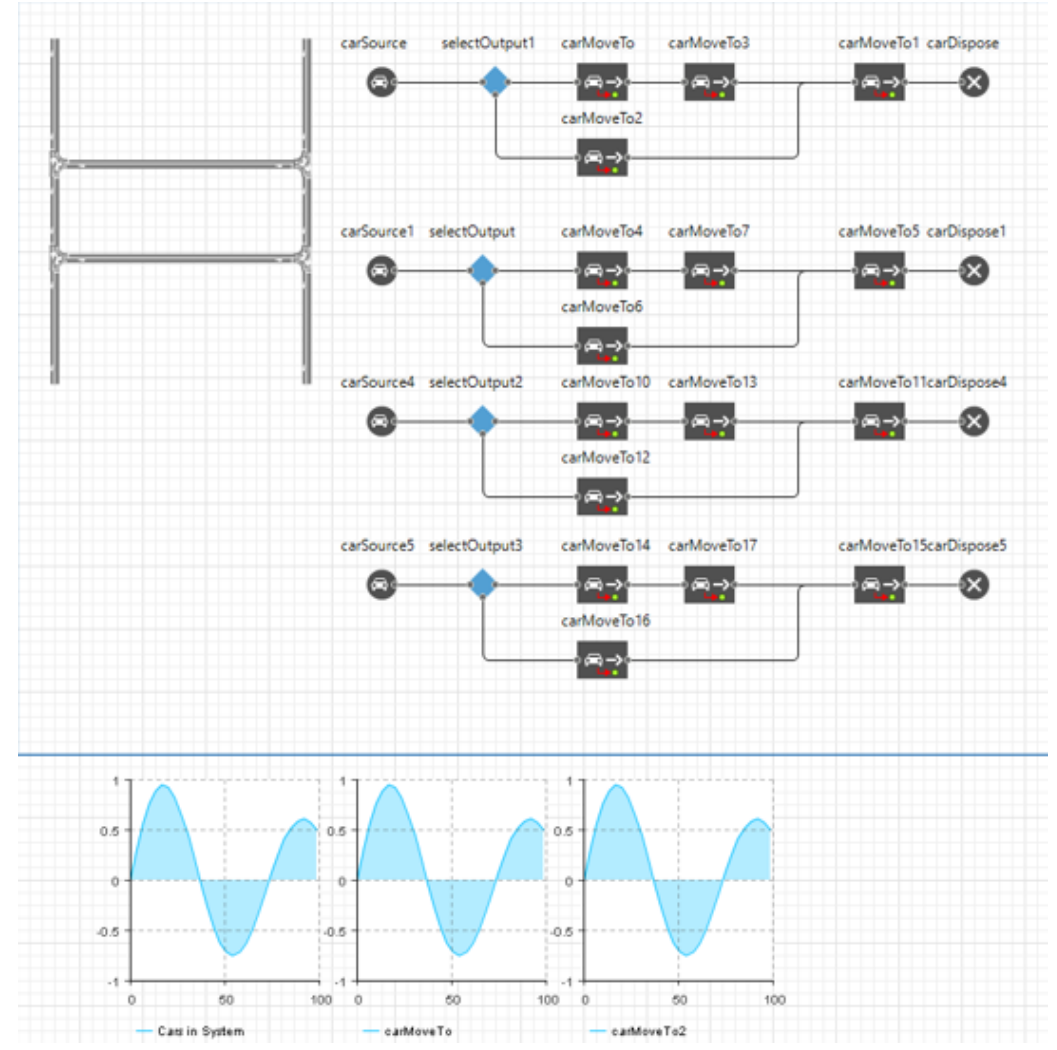

Fig. 1 Algorithm logic. Source: authors 
In the next step, a simple condition is inserted into the "selectOutput" blocks, which, depending on the number of vehicles in a given turn, decide which way the vehicles should continue on. Table 1 shows the hourly traffic intensities in relation to the average time spent in the simulation using the system of adaptive junction control with respect to an uncontrolled junction. The values in the table were obtained via the "histogram" function and via the "timeInModel" command (time spent in the simulation) and the "mean" function (average value for all agents in the simulation).

Table 1 Comparison of Controlled and Uncontrolled Intersections. Source: authors

\begin{tabular}{ccccccccccccc}
\hline $\begin{array}{c}\text { Traffic } \\
\text { intensity } \\
\text { [veh/hrs] }\end{array}$ & 1000 & 1100 & 1200 & 1300 & 1400 & 1500 & 1600 & 1800 & 2000 & 2200 & 2400 & 2600 \\
\hline $\begin{array}{c}\text { Route time } \\
\text { in model } \\
\text { mean - } \\
\text { Controlled } \\
{[\mathrm{s}]}\end{array}$ & 51.34 & 49.54 & 50.67 & 54.8 & 55.12 & 55.87 & 56.90 & 59.32 & 62.40 & 67.97 & 93.25 & 115.0 \\
\hline $\begin{array}{c}\text { Route time } \\
\text { in model } \\
\text { mean - not } \\
\text { controlled } \\
{[\mathrm{s}]}\end{array}$ & 42.15 & 43.78 & 43.86 & 43.7 & 45.73 & 55.77 & 59.61 & 63.95 & 68.92 & 75.81 & 117.5 & 218.9 \\
\hline $\begin{array}{c}\text { Share of } \\
\text { values [\%] }\end{array}$ & 82.09 & 88.38 & 86.54 & 79.6 & 82.97 & 99.81 & 104.7 & 107.7 & 110.4 & 111.5 & 126.0 & 190.2 \\
\hline
\end{tabular}

The graph in Fig. 4 shows a comparison of the time spent in the simulation in the case of using an adaptive junction control system and in the case of using an uncontrolled junction. The graph below shows that the use of an adaptive junction management system is advantageous only from a certain level of traffic density, in this case study it was a value higher than 1500 agents per hour.

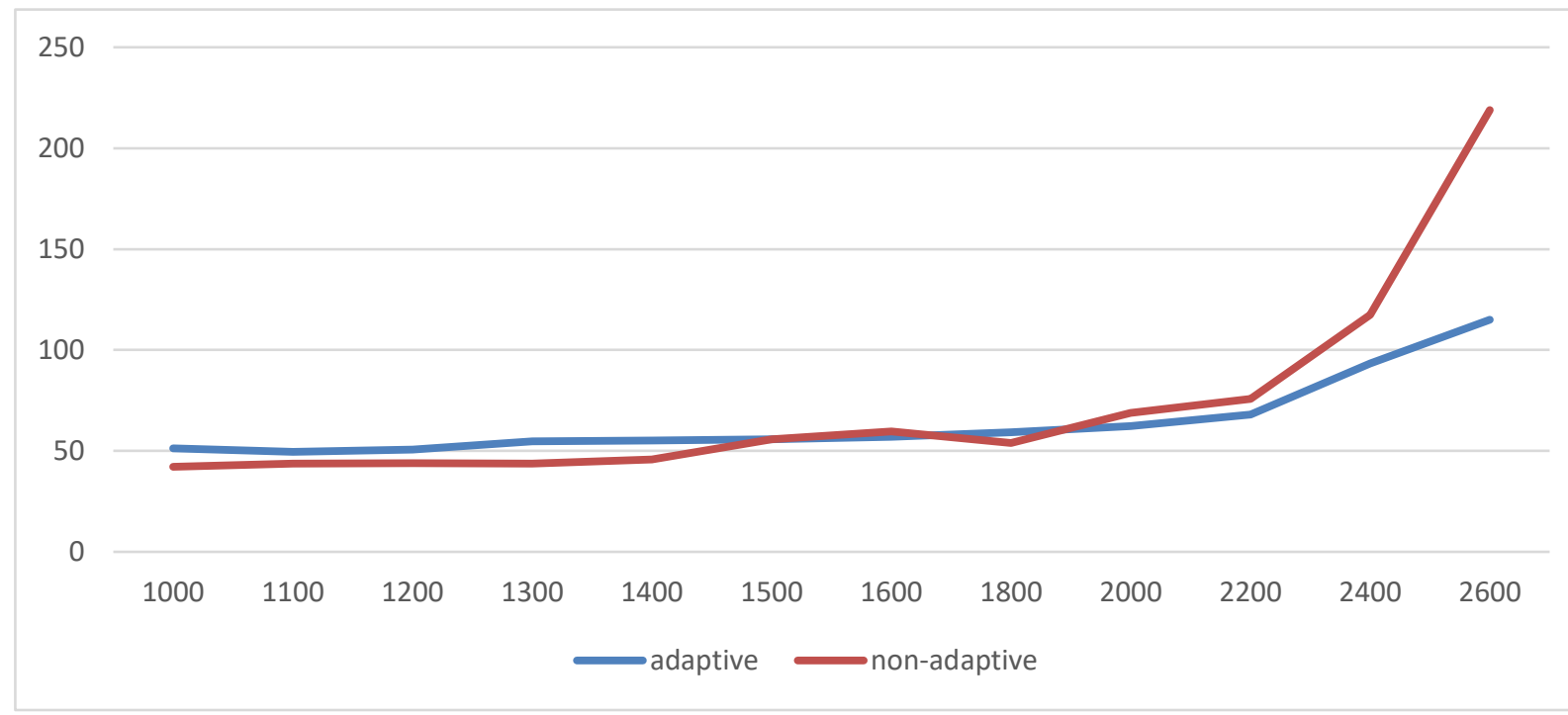

Fig. 2 Comparison of time spent in simulation in controlled and uncontrolled intersection. Source: authors 


\section{Conclusion}

Using the AnyLogic simulation software, this case study reduced the average time spent in the simulation as the main goal of the optimization experiment. In the context of autonomous mobility and "Connected Vehicles" technology, this procedure can be used not only to optimize controlled intersections in real time based on current numbers of vehicles on a given section, but also future (prospective) values combined with the possibility of determining the optimal route according to the number of vehicles on the given section at a given moment in time. In addition to the above, it is also possible to use different types of agents and their preferences when passing through the transport network or automatically divert traffic from the desired section, which can simulate the passage of integrated rescue systems, public transport vehicles or other crisis situations. The increase in traffic throughput in the simulation of autonomous mobility and the "Connected Vehicles" technology was 5 to $20 \%$, depending on the specific situation in the locality in question, which was the subject of this case study. Given that general optimization will lead to a reduction in the density and volume of traffic in a given locality, another benefit of autonomous driving will also be a reduction in the energy intensity of transport and greenhouse gas emissions, which is currently a very topical issue.

\section{Acknowledgments}

Research outputs presented in the paper were funded within the specific scientific project at the Institute of Technology and Business in Ceske Budejovice named "Designing of a system enabling long-term operation of unmanned aerial vehicles for the purpose of traffic monitoring" (SVV202106).

\section{References}

[1] Guler, S.I., Menendez, M. \& Meier, L. (2014). Using Connected Vehicle Technology to Improve the Efficiency of Intersections. Transportation Research Part C: Emerging Technologies. 46, 121-131. DOI: 10.1016/j.trc.2014.05.008.

[2] Maslekar, N., Mouzna, J. Boussedjra, M. \& Labiod, H. (2013). CATS: An Adaptive Traffic Signal System Based on Car-to-Car Communication. Journal of Network and Computer Applications 36(5), 1308-1315. DOI: 10.1016/j.jnca.2012.05.011.

[3] Younes, M.B. \& Boukerche, A. (2016). Intelligent Traffic Light Controlling Algorithms Using Vehicular Networks. IEEE Trans Veh. Technol. 65, 5887-5899. DOI:10.1109/TVT.2015.2472367.

[4] Nafi, N.S. \& Khan, J.Y. (2012). A VANET Based Intelligent Road Traffic Signalling System. In Proceedings of the Telecommunication Networks and Applications Conference, 7-9 November 2012, Brisbane, QLD, Australia. DOI:10.1109/ATNAC.2012.6398066. 
[5] Jennings, N.R., Sycara, K. \& Wooldridge, M.A. (1998). Roadmap of Agent Research and Development. Auton. Agents Multi-Agent Syst. 1998, 1, 7-38. DOI:10.1023/A:1010090405266.

[6] Kari, D., Wu, G. \& Barth, M.J. (2014). Development of an Agent-based Online Adaptive Signal Control Strategy Using Connected Vehicle Technology. In Proceedings of the IEEE International Conference on Intelligent Transportation Systems, 8-11 October 2014, Qingdao, China. DOI:10.1109/ITSC.2014.6957954.

[7] Ezawa, H. \& Mukai, N. (2010). Adaptive Traffic Signal Control Based on Vehicle Route Sharing by Wireless Communication; Springer: Berlin/Heidelberg, Germany, 280-289. DOI:10.3390/info8030101.

[8] Xiang, J. \& Chen, Z. (2016). An Adaptive Traffic Signal Coordination Optimization Method Based on Vehicle-to-Infrastructure Communication. Cluster Comput., 19, 1-12. DOI:10.1007/s10586-016-0620-7.

[9] Caban, J., Droździel, P., Krzywonos, L., Rybicka, I.K., Šarkan, B. \& Vrábel, J. (2019). Statistical Analyses of Selected Maintenance Parameters of Vehicles of Road Transport Companies. Advances in Science and Technology Research Journal, 13(1), 1-13. DOI: $10.12913 / 22998624 / 92106$.

[10] Lizbetin, J., Stopka, O. \& Kurenkov, P.V. (2019). Declarations Regarding the Energy Consumption and Emissions of the Greenhouse Gases in the road Freight Transport Sector. The Archives of Automotive Engineering - Archiwum Motoryzacji, 83(1), 59-72. DOI: 10.14669/AM.VOL83.ART4.

[11] Fedorko, G., Heinz, D., Molnár, V. \& Brenner, T. (2020). Use of Mathematical Models and Computer Software for Analysis of Traffic Noise. Open Engineering, 10(1), 129-139. DOI: 10.1515/eng-2020-0021.

[12] Jurkovič, M., Kalina, T., Skrúcaný, T., Gorzelańczyk, P. \& Lupták, V. (2020). Environmental Impacts of Introducing LNG as Alternative Fuel For Urban Buses - Case Study in Slovakia. Promet - Traffic - Traffico, 32(6), 837-847. DOI:10.7307/ptt.v32i6.3564.

[13] Maghrour Zefreh, M. \& Török, A. (2020). Distribution of Traffic Speed in Different Traffic Conditions: An Empirical Study in Budapest. Transport 35.1, 68-86. DOI: $10.3846 / 2019.11725$

[14] Stopka, O., Sarkan, B., Chovancova, M. \& Kapustina, L.M. (2017). Determination of the Appropriate Vehicle Operating in Particular Urban Traffic Conditions. Communications 
Scientific Letters of University of Zilina 19.2, 18-22. Retrieved October, 11, 2021, from http://komunikacie.uniza.sk/index.php/communications/article/view/176

[15] Lupták, V., Hlatká, M. \& Kampf, R. (2018). Energy Consumption and Greenhouse Gases Emissions on Relation Brno-Jihlava. Paper presented at the MATEC Web of Conferences, 235,3-9. DOI: 10.1051/matecconf/201823500011.

[16] To, C.N., Milani, S., Marzbani, H. \& Jazar, R. N. (2021). Improvement of the Autodriver Algorithm for Autonomous Vehicles Using Roll Dynamics. The Archives of Automotive Engineering - Archiwum Motoryzacji, 91(1), 5-23. DOI: 10.14669/AM.VOL91.ART1.

[17] Stopka, O., Zitricky, V., Abramovic, B., Marinov, M. \& Ricci, S. (2019). Innovative Technologies for Sustainable Passenger Transport. Journal of Advanced Transportation, vol. 2019, article ID 4197246, 2 pages, DOI: 10.1155/2019/4197246.

[18] Török, Á., Szalay, Z., Uti, G. \& Verebélyi, B.(2020). Rerepresenting Autonomated Vehicles in a Macroscopic Transportation Model. Periodica Polytechnica Transport Engineering 48.3, 269275. ISSN 2352-1465.

[19] Caban, J. (2021). Study of eco-driving possibilities in passenger car used in urban traffic. The Archives of Automotive Engineering - Archiwum Motoryzacji 91(1), 37-48. DOI: 10.14669/AM.VOL91.ART3.

[20] Zitrický, V., Gašparík, J. \& Pečený, L. (2015). The Methodology of Rating Quality Standards in the Regional Passenger Transport. Transport Problems 10, 59-72. DOI: 10.21307/tp-2015062. 